\title{
Directional excitation of surface plasmons by dielectric resonators
}

\author{
Chengjun Zou, ${ }^{1}$ Withawat Withayachumnankul, ${ }^{1}$ Ilya V. Shadrivov, ${ }^{2}$ Yuri S. Kivshar, ${ }^{2}$ and Christophe Fumeaux ${ }^{1, *}$ \\ ${ }^{1}$ School of Electrical \& Electronic Engineering, The University of Adelaide, Adelaide, SA 5005, Australia \\ ${ }^{2}$ Nonlinear Physics Centre, The Australian National University, Canberra, ACT 2601, Australia \\ (Received 9 December 2014; revised manuscript received 9 February 2015; published 27 February 2015)
}

\begin{abstract}
An important aim of current research on plasmonics is to develop compact components to manipulate surface plasmon polaritons (SPPs) and specifically to develop efficient SPP couplers. The commonly used metallic resonators are inefficient to couple free-space waves to SPPs and metallic gratings require oblique incidence for achieving unidirectional propagation. In this article, we propose to use nanoscale nonuniform arrays of dielectric resonator antennas (DRAs) to realize unidirectional launching of SPPs. DRAs are made of low-loss high-permittivity nanostructures operating on a metal surface. The applications of metallodielectric nanostructures can produce resonances mainly in the low-loss dielectric parts and hence the power dissipated through oscillating current in metal can be reduced. Similar to metallic resonators, DRAs operating near resonance can provide phase control when coupling incident waves into SPPs, adding degrees of freedom in controlling propagation direction. The theoretical analysis in this article, with numerical validation, shows efficient SPPs launching by nonuniform array of cylindrical DRAs into a predesigned direction. Furthermore, with proper patterning, optimal launching can be achieved by avoiding power leakage via deflection into free space. The SPP launching condition and the influence of propagation loss are also mathematically analyzed from the viewpoint of antenna array theory. The SPPs launchers based on DRAs have a potential for applications in highly efficient integrated optics and optical waveguides.
\end{abstract}

DOI: 10.1103/PhysRevB.91.085433

PACS number(s): 73.20.Mf

\section{INTRODUCTION}

Surface plasmon polaritons (SPPs) are propagating electromagnetic waves that are confined along metal-dielectric interfaces and are coupled with collective electron oscillations [1]. Due to their subwavelength nature and spatially confined field energy, SPPs can localize guided waves beyond the diffraction limit, and thus their exploitation is promising for applications in integrated optics [2,3], field enhancement [4-6], sensing $[7,8]$, and imaging $[9,10]$. A major pursuit of current plasmonics research is to develop compact and efficient components to manipulate SPP propagation. Because SPP cannot be excited directly by incident light from free space, efficient SPP couplers are important components. In particular, the function of unidirectionally launching SPP can be applied to integrated optics. Prisms and metal gratings are conventionally used to couple free-space propagating waves to SPPs [1]. However, prisms are too bulky for integrated optics, while metallic gratings based on Wood's anomalies [11] require phase differences introduced by oblique incidence to realize unidirectional launching [12].

So far, different nanoscale structures have been demonstrated for SPP unidirectional launching, aiming at overcoming the limitations of the conventional coupling methods and providing discrete components for integrated optics. A conventional nanoslit on a metal surface, combined with metallic gratings [13] or Bragg mirrors [14,15], can guide or reflect SPPs to a desired direction. With some modification, a single asymmetric nanoslit [16] can realize unidirectional SPP launching based on wave interference from the Fabry-Pérot nanocavity. Single grooves [17] and holes [18] on metallic

*Electronic address: cfumeaux@eleceng.adelaide.edu.au surfaces have also been demonstrated for SPP coupling. By changing the dimensions of grooves or holes, a phase shift can be added to the excited SPPs. Thus a nonuniform array of grooves [19,20] and holes [21] of varying dimensions can achieve unidirectional SPP launching resulting from wave interference. In a sophisticated realization, metallic slits have been demonstrated as polarization-dependent SPP launchers, where normally incident waves with orthogonal polarizations result in opposite SPP launching directions [22]. Recently, Liu et al. presented a compact magnetic antenna launcher [23] consisting of two near resonant $\mathrm{Au}-\mathrm{MgF}_{2}-\mathrm{Au}$ sandwich structures. This arrangement is equivalent to two horizontal magnetic dipoles where a phase shift is obtained from different antenna dimensions. Other interesting unidirectional SPP launchers such as a structure mimicking a single rotating dipole [24] and broadband couplers [25] have been demonstrated on the basis of the optical antenna concept $[26,27]$.

In this article, we propose an alternative nanoantenna element with coupling properties suitable for unidirectional SPP launching. The proposed launching structures are based on arrays of nonuniform cylindrical dielectric resonator antennas (DRAs) on a metallic plane, as shown in Fig. 1(a). Dielectric resonators in varying sizes provide an accurate localized control of excited SPP phases. The proposed realization in the form of a nonuniform array offers additional degrees of freedom for efficient SPP coupling and launching. Moreover, optimal launching conditions are investigated in this article, leading to design guidelines for nonuniform arrays of subwavelength resonators. Simulation results demonstrate how an optimal coupling is found in a nondeflection zone where the first diffraction order is prohibited so that the incident power can be predominantly coupled into SPPs. 


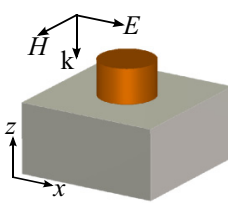

(a)

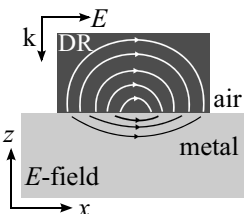

(b)

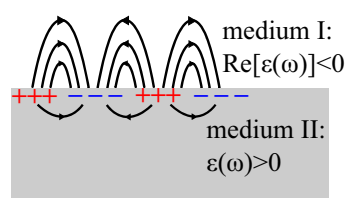

(c)
FIG. 1. (Color online) (a) A 3D illustration shows a cylindrical dielectric resonator (DR) on a metal surface. (b) The illustration of electric field distribution in the $x z$-plane cross-section. (c) Illustration of typical $E$-field distribution of SPPs along the dielectric-conductor interface.

\section{SPP LAUNCHING BASED ON DRAS}

Dielectric resonator antennas have a wide range of applications at microwave frequencies [28]. Operated in their fundamental modes, DRAs usually have a compact size, low loss, and moderate $Q$ factor [29]. Different from conventional metallic antennas, DRAs have been demonstrated to keep their high efficiency [30-32] even in the optical range [33]. The proposed SPP launching structure consists of an array of nonuniform cylindrical DRAs on a metal substrate, for which the dimensions of the elements are varied only in one direction. The array is excited with TM polarized light, such that the $E$-field of the incident wave is aligned with the direction in which the array is nonuniform. Under this excitation condition, resonant DRAs are intended to operate in their fundamental $\mathrm{HEM}_{11 \delta}$ mode, which has a field distribution shown in Fig. 1(b). The electric field of the cylindrical DRA in this $\mathrm{HEM}_{11 \delta}$ mode circulates perpendicularly to the metal surface, and the magnetic field oscillates in the DRA center in parallel to the substrate surface. This field distribution corresponds to that of a horizontal magnetic dipole on a ground plane. The feature of the $\mathrm{HEM}_{11 \delta}$ field distribution resembles the SPPs field, shown in Fig. 1(c), indicating the potential of DRAs for efficient SPP coupling. Hence, low-loss DRAs operating at optical frequencies appear as an ideal building block for efficient and compact SPP couplers. Different from the metal grating coupling, nonuniform DRAs can introduce tailored phase shifts among excited SPPs. With proper arrangement, a nonuniform array of DRA can asymmetrically give rise to constructive and destructive interference in opposite directions along the metal surface and thus unidirectionally launch SPPs.

\section{OPTIMAL SPP LAUNCHING}

Based on the SPP-coupling mechanism with DRAs introduced in Sec. II, we further analyze optimal launching conditions. This analysis is not limited to structures proposed in this article. For simplification of the theoretical considerations, the DRAs of different sizes are initially modeled as ideal point sources with identical radiative loss and varying phases, while SPP propagation is assumed lossless. Under these assumptions, optimal launching conditions of SPPs are defined in Sec. III A for both normal and oblique incidence. A more detailed analysis of the DRA-based launcher considering losses is then presented in Sec. III B.

\section{A. Optimal launching condition}

A common principle for unidirectional launching of SPPs is based on the wave interference. This interference process is illustrated in Fig. 2(a) for the proposed SPP launcher based on DRAs. For simplicity, only two adjacent DRAs from an infinite array are considered first. The DRAs are located on a silver surface with a distance $d$ between their centers and they have phase response on reflection corresponding to $\phi_{1}$ and $\phi_{2}$. Illuminated by a TM polarized light with an incidence angle $\theta_{i}$, the two resonators couple the incident waves into SPPs. The SPPs excited by those two elements have phase differences imposed by the elements' intrinsic phase responses, the incident angle, and the different propagation distances.

With proper phase matching, constructive interference can be achieved on one side along the metal surface, with destructive interference on the other side. This phase matching condition can be generally described as

$$
\phi_{1} \pm k_{\mathrm{spp}} d=\phi_{2}+k_{0} d \sin \theta_{i}+2 v \pi \text {. }
$$

In Eq. (1), $k_{0}$ is the wavenumber of the incident free-space wave and $k_{\text {spp }}$ denotes the wavenumber of the propagating SPPs launched by the DRAs. The case of "+" in Eq. (1) represents perfect constructive interference toward the right-hand side while the "-" describes the perfect constructive interference toward the left-hand side. The amplitude of the wavevector $\boldsymbol{k}_{\text {spp }}$ is defined with positive sign to the right as shown in Fig. 2(a). The integer $v$ denotes the SPP launching order. Under normal

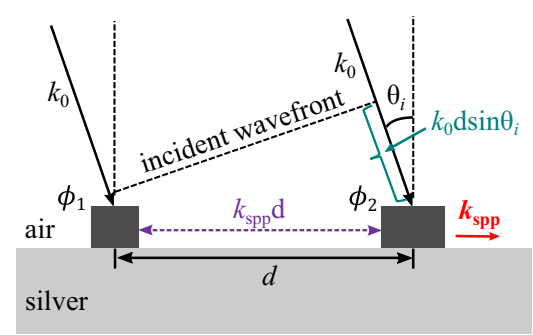

(a)

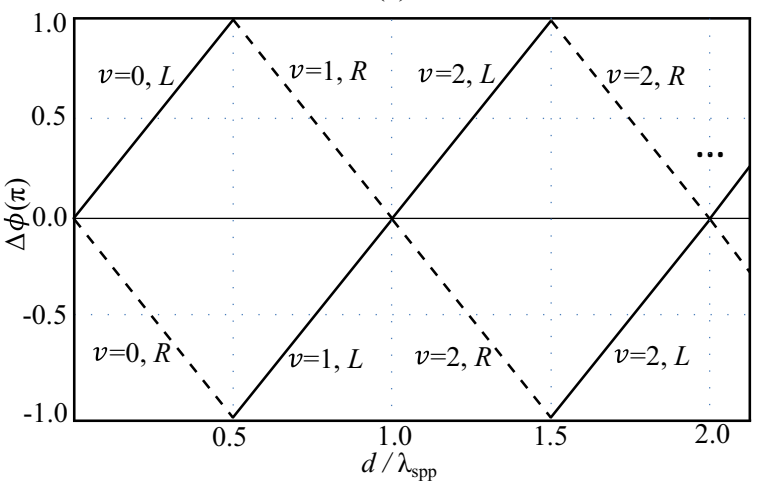

(b)

FIG. 2. (Color online) DRA array for SPP excitation: (a) Schematic of DRAs for SPP unidirectional launching. (b) SPP directional launching lines for an infinite ideal array of DRAs. Here $v$ denotes the diffraction order while $L$ and $R$ denote left (solid line) and right (dashed line) directions. 


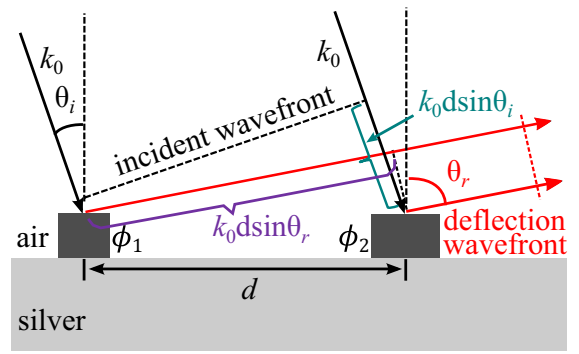

(a)

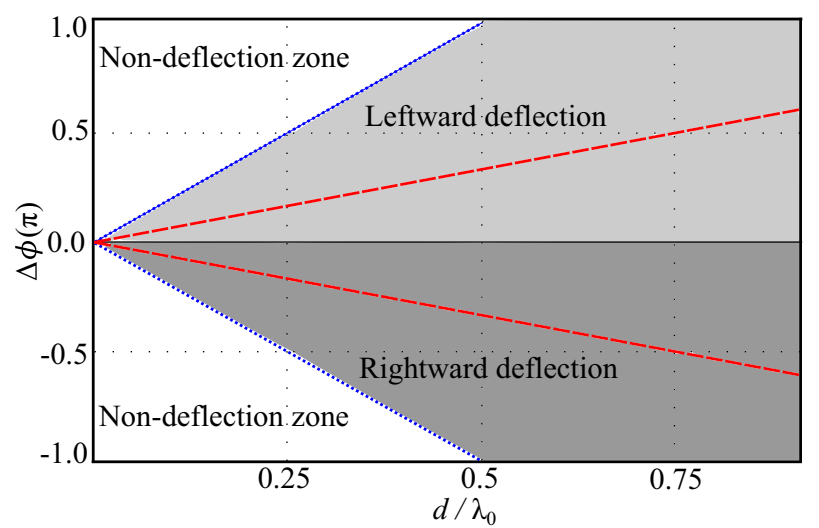

(b)

FIG. 3. (Color online) DRA array in reflectarray configuration: (a) Schematic of DRAs for reflectarray beam deflection. (b) Deflection zone and nondeflection zone. The blue dotted lines and red dashed lines denote the first- and second-order deflections tangential to the surface $\left(\theta_{r}= \pm 90^{\circ}\right)$, respectively.

incidence, where $\theta_{i}=0^{\circ}$, Eq. (1) is simplified to

$$
\pm k_{\mathrm{spp}}=\frac{\Delta \phi}{d}+v \frac{2 \pi}{d},
$$

with $\Delta \phi=\phi_{2}-\phi_{1}$. The solutions of Eq. (2) is graphically presented in Fig. 2(b). The plotted lines indicate perfect constructive interference on one side but destructive interference on the other side. Practically, lower launching orders $v$, and small distances $d$ are preferred to minimize propagation losses. This preference is also reflected in the analysis in Sec. III B.

Besides SPP launching, the progressive phase response of a nonuniform DRA array can be configured for realization of reflectarrays at optical frequencies [34,35]. The optical reflectarray in Ref. [34] was demonstrated to deflect incident light into a predesigned direction in free space. In the context of SPP launching, this deflection mechanism reduces the power available for surface-wave coupling and must be suppressed. In the following paragraph within Sec. III A, we analyze how to minimize power leakage from deflection and achieve optimal SPP launching.

As illustrated in Fig. 3(a), the beam deflected by two adjacent elements toward an angle $\theta_{r}$ can be described by the phase matching condition:

$$
\phi_{1}+k_{0} d \sin \theta_{r}=\phi_{2}+k_{0} d \sin \theta_{i}+2(m-1) \pi .
$$

Here the index $m=1,2,3 \ldots$ represents diffraction orders created by the two adjacent resonators. The numbering " $m-1$ " is selected such that $m=1$ matches the first diffraction order. For normal incidence $\left(\theta_{i}=0^{\circ}\right)$, the $m$ th-order diffraction

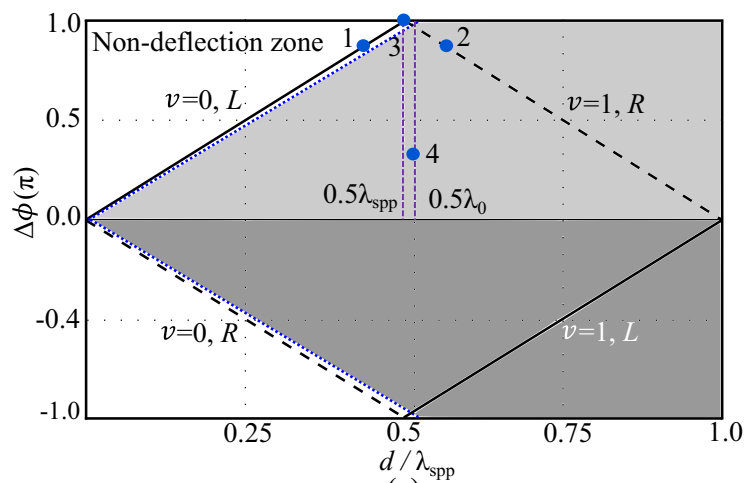

(a)

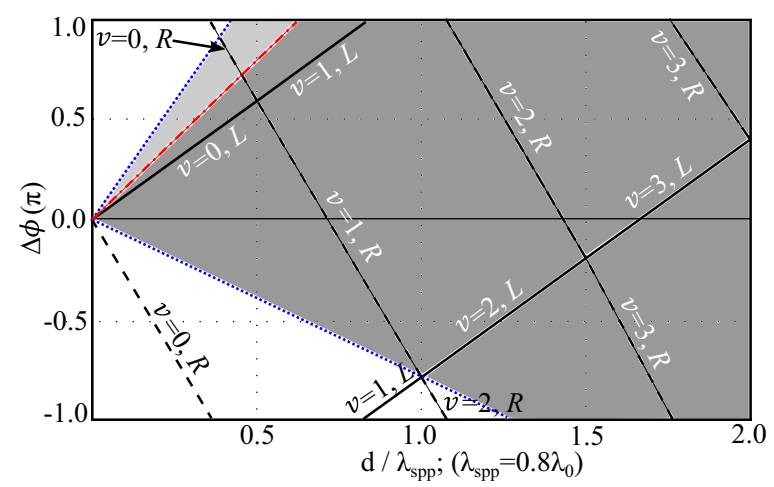

(b)

FIG. 4. (Color online) SPP launching condition: (a) Normal incidence with $k_{\mathrm{spp}}=1.033 k_{0}$ (SPP wavenumber calculated on silver-air interface at $633 \mathrm{~nm}$ ). The blue dots 1, 2, and 3 correspond to design and simulation cases 1,2 , and 3 , respectively, in Sec. IV. The reflectarray realized in Ref. [34] is also labeled as dot 4. (b) Oblique incidence at $30^{\circ}$ with increased $k_{\mathrm{spp}}=1.25 k_{0}$ for illustration purposes.

(deflection) angle can be calculated as

$$
\sin \theta_{r}=\frac{\Delta \phi}{k_{0} d}+(m-1) \frac{2 \pi}{k_{0} d} .
$$

For the array configuration with progressive $\Delta \phi$ and interelement spacing $d$, the $m=1$ order deflection is the most relevant and needs to be suppressed. From Eq. (4), a necessary condition can be found by considering that the $m=1$ order deflection can be accessed only when $\left|\sin \theta_{r}\right| \leqslant 1$; i.e.,

$$
\frac{|\Delta \phi|}{d} \leqslant k_{0}
$$

This condition is presented graphically in Fig. 3(b), showing the well-defined areas as deflection zones (shaded; $\left|\sin \theta_{r}\right| \leqslant$ 1) and nondeflection zones (unshaded; $\left|\sin \theta_{r}\right|>1$ ). To facilitate the analysis of achieving optimal SPP launching and suppressing wave deflection, Figs. 2(b) and 3(b) are combined into Fig. 4(a), in which $\lambda_{0}$ is selected to be $633 \mathrm{~nm}$ (equivalent to $474 \mathrm{THz}$ ) and the corresponding $\lambda_{\text {spp }}$ is calculated on a silver-air interface. It is seen in Fig. 4(a) that the zeroth-order directional SPP launching lines lie inside the nondeflection zone. Hence, the lines satisfy the condition for unidirectional SPP launching, while prohibiting power leakage from deflection. The $(\Delta \phi, d)$ pairs corresponding to these 
lines fulfill

$$
\frac{|\Delta \phi|}{d}=k_{\mathrm{spp}}>k_{0}
$$

Therefore, any pair along the $v=0$ order lines promises high SPP launching efficiencies while the crossing points between the $L$ and the $R$ lines such as $\left(\frac{\lambda_{\text {spp }}}{2}, \pi\right)$ promises bidirectional launching, which, in the case of infinite arrays, will result in standing waves. The nondeflection condition introduced in this section defines a radiation pattern with a unique main lobe along the launching direction on the metal surface, and without grating lobes to match higher diffraction orders [36]. In effect, optimal SPP launching can be achieved.

The considerations of optimal launching can be extended to the case of oblique incidence, where the cumulative phase shift is caused by both the resonators' phase responses and the incidence angle. For this general case, Eq. (1) can be used for describing the ideal SPPs momentum matching, while the deflection conditions can be retrieved from Eq. (3). In particular, for the $m=1$ order, the deflection angle can be calculated as

$$
\sin \theta_{r}=\frac{\Delta \phi}{k_{0} d}+\sin \theta_{i} .
$$

To illustrate the effect of the angle of incidence, a sample case with an incidence angle $\theta_{i}=30^{\circ}$ is presented in Fig. 4(b) where the $k_{\mathrm{spp}}$ is increased to $1.25 k_{0}$ for better illustration. It is observed that an asymmetry is introduced by the incidence angle and both the deflection zone and directional propagation lines are rotated in the graph. Nevertheless, optimal launching condition can be identified on the SPP launching line in the nondeflection zone, where the deflection angle $\theta_{r}$ becomes imaginary. Thus, the optimal condition can be described as

$$
\frac{|\Delta \phi|}{d}+k_{0} \sin \theta_{i}=k_{\mathrm{spp}}>k_{0} .
$$

The red dash-dotted line in Fig. 4(b) denotes the Littrow configuration where the incident beam is reflected back along the incident path [37].

\section{B. Linear array theory for SPP launching}

The analysis in Sec. III A offers general guidelines for designing optimal launchers in an ideal infinite array based on the interelement distance $d$ and the progressive phase shift $\Delta \phi$. However, this analysis does not include the effects of SPP propagation loss and of the finite extent of the array. Here we extend this analysis with a simple one-dimensional interference model that assumes a finite $N$-element array repeated infinitely in the direction perpendicular to the SPP launching direction. Additionally, all DRAs, excited by a normal incident TM-polarized light, are assumed to have the same radiation pattern and the uniform amplitude of unity. As shown in Fig. 5, the $N$-resonator array is located on a silver surface and the elements have a progressive phase shift of $\Delta \phi$. According to the interference principle, the total amplitude is the superposition of SPPs launched by all the resonators to either sides of the array. The total amplitude of the SPPs at observation points located at a distance $D$ from the edge resonator on the left and right can be calculated as (with the

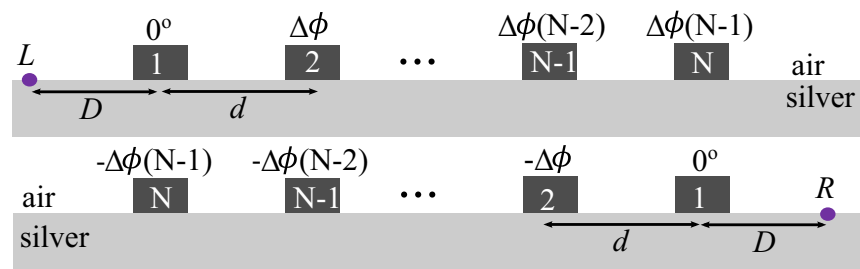

FIG. 5. (Color online) Concept of a $N$-DRA array with progressive phase. The observation points $L$ and $R$ are set at left and right sides of the array.

time dependence $e^{i \omega t}$ omitted)

$$
\begin{aligned}
A_{L} & =\sum_{n=1}^{N} e^{-i k_{\mathrm{spp}}[D+(n-1) d]+i(n-1) \Delta \phi} \\
& =e^{-i k_{\mathrm{spp}} D} \frac{1-e^{i N\left(\Delta \phi-k_{\mathrm{spp}} d\right)}}{1-e^{i\left(\Delta \phi-k_{\mathrm{spp}} d\right)}} ; \\
A_{R} & =\sum_{n=1}^{N} e^{-i k_{\mathrm{spp}}[D+(n-1) d]-i(n-1) \Delta \phi} \\
& =e^{-i k_{\mathrm{spp}} D} \frac{1-e^{i N\left(\Delta \phi+k_{\mathrm{spp}} d\right)}}{1-e^{i\left(\Delta \phi+k_{\mathrm{spp}} d\right)}} .
\end{aligned}
$$

In Eq. (9), $A_{L}$ and $A_{R}$ are the total SPP amplitude summations for propagation toward the left and right sides, respectively. The complex SPP wavenumber $k_{\text {spp }}$ includes the phase constant $\beta$ and the attenuation constant $\alpha$. Provided that the array operates at $633 \mathrm{~nm}$ on a silver-air interface, the complex SPP wavenumber is $k_{\mathrm{spp}}=1.025 \times 10^{7}-i 1.017 \times$ $10^{4} \mathrm{rad} / \mathrm{m}[1,34]$. In the following, we will analyze SPP launching in this formalism starting from the infinite ideal array and later increasing complexity to observe the impact of the finite extent and propagation losses on the launching performance.

First, the propagation loss is neglected or equivalently $k_{\mathrm{spp}}$ is assumed real. For an infinite array, i.e., the number of resonators $N=\infty$, the normalized left and right amplitudes can be analytically calculated as

$$
\begin{aligned}
A_{L} / N & =\lim _{N \rightarrow \infty} \frac{1}{N} \frac{1-e^{i N\left(\Delta \phi-k_{\mathrm{spp}} d\right)}}{1-e^{i\left(\Delta \phi-k_{\mathrm{sp}} d\right)}} \\
& = \begin{cases}1 ; & \Delta \phi=k_{\mathrm{spp}} \cdot d+2 m \pi \\
0 ; & \text { otherwise, }\end{cases} \\
A_{R} / N & =\lim _{N \rightarrow \infty} \frac{1}{N} \frac{1-e^{i N\left(\Delta \phi+k_{\mathrm{spp}} d\right)}}{1-e^{i\left(\Delta \phi+k_{\mathrm{spp}} d\right)}} \\
& = \begin{cases}1 ; & \Delta \phi=-k_{\mathrm{spp}} \cdot d+2 m \pi \\
0 ; & \text { otherwise, }\end{cases}
\end{aligned}
$$

where $m$ is an arbitrary integer. This analysis confirms that for infinite lossless arrays, optimal directional launching is satisfied when $\Delta \phi=k_{\mathrm{spp}} d+2 m \pi$, i.e., on directional launching lines shown in Fig. 2(b). On the other hand, if the condition is not strictly satisfied, the SPP vanishes.

For a finite array, optimal launching is still achieved by arrays on the directional launching lines in Fig. 2(b), but other 


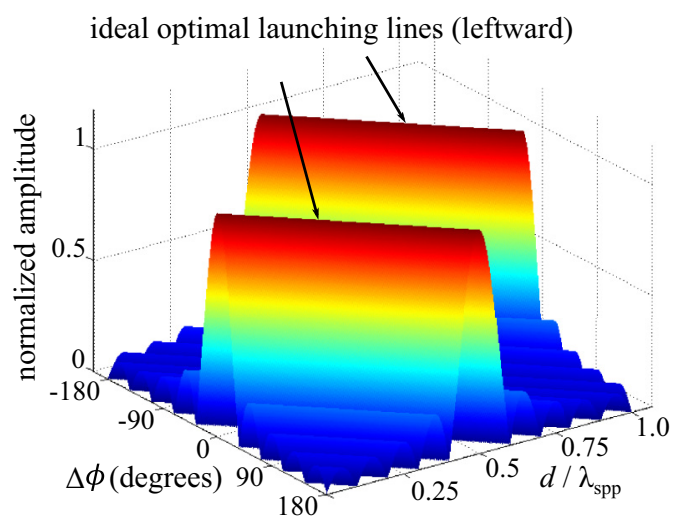

FIG. 6. (Color online) Normalized SPP amplitude toward the left-hand side of finite arrays $(N=10)$ with different $d$ and $\Delta \phi$.

array configurations that are not located on these lines can also lead to nonzero SPP amplitude. This is exemplified in Fig. 6 for propagation toward the left, in the case $N=10$. The difference between normalized launched power to the left side and right side is presented in Fig. 7. The result from Fig. 7 confirms that the difference of launched power

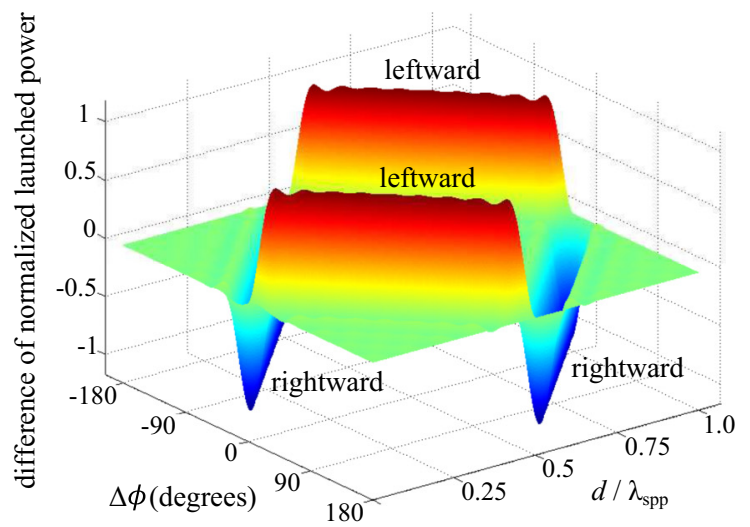

(a)

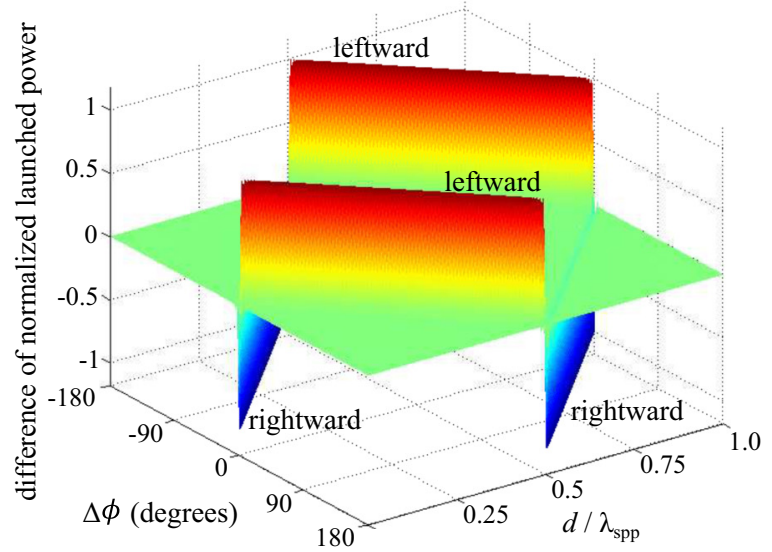

(b)

FIG. 7. (Color online) Difference of normalized power launched to the left (positive) and right (negative) for values (a) $N=10$ and (b) $N=100$.

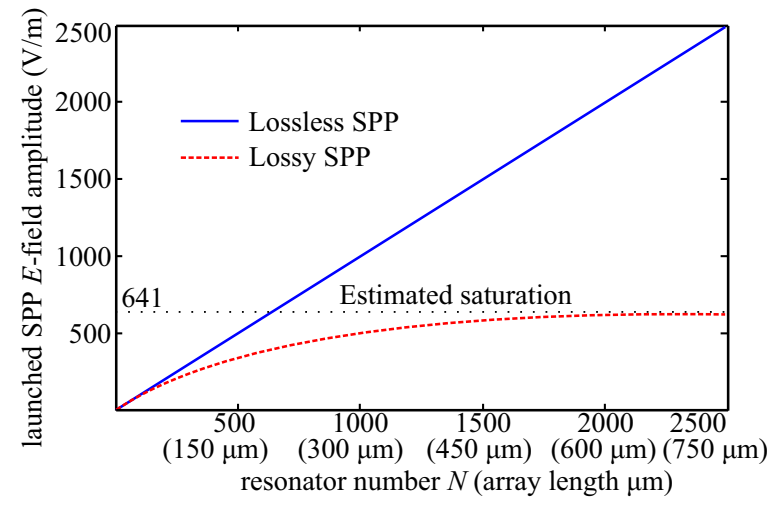

FIG. 8. (Color online) Launched SPPs $E$-field amplitude for lossy (red) and lossless (blue) conditions, calculated at silver-air interface at $633 \mathrm{~nm}$. Each resonator is assumed to have SPP amplitude of $1 \mathrm{~V} / \mathrm{m}$.

from finite arrays in the nonoptimal launching region is not strictly zero and this effect is more obvious for smaller arrays than for larger arrays. With an increased number of resonators, the regions of unidirectional launching become increasingly narrower and, as can be expected, the results rapidly converge to the launching lines when $N$ approaches infinity. It is also observed that on the launching lines, the SPP power for finite arrays is not constant, since a small amount of power can be launched toward the opposite direction too.

Now the analysis is extended to include the impact of the propagation loss by considering the complex SPP wavenumber $k_{\text {spp }}$ given earlier. At $633 \mathrm{~nm}$, the one-dimensional propagation length [38] of SPPs (with infinite transversal extent) on an ideally flat silver-air interface is approximately equal to $50 \mu \mathrm{m}[1,34]$, where the energy decays along the propagation direction of SPPs wavefronts to $1 / e$ or the amplitude decays to about $60.6 \%$. Due to the amplitude attenuation, SPPs excited by a given resonator have negligible contribution to the total SPP beyond a certain distance. Mathematically, a reexamination of Eq. 9(a), with $k_{\mathrm{spp}}$ replaced by $\beta-i \alpha$, yields

$$
A_{L, \alpha}=e^{-i k_{\mathrm{spp}} D} \cdot \frac{1-e^{i N(\Delta \phi-\beta d)} e^{-N d \alpha}}{1-e^{i(\Delta \phi-\beta d)} e^{-d \alpha}},
$$

where $A_{L, \alpha}$ is the launched SPP amplitude at a distance $D$ away from a $N$-element array with given interelement distance $d$ and progressive phase $\Delta \phi$. Due to the propagation loss, it can be indeed calculated that the value of $A_{L, \alpha}$ converges to $e^{-i k_{\mathrm{spp}} D} /\left(1-e^{i(\Delta \phi-\beta d)} e^{-\alpha d}\right)$ when $N$ approaches infinity and $e^{-N d \alpha}$ becomes zero. An example, corresponding to the $(\Delta \phi$, d) pair $\left(\frac{\pi}{2}, \frac{\lambda_{\text {spp }}}{4}\right)$ on $v=0$ ( $L$ line $)$ is presented in Fig. 8 , where it is clearly visible that the launched SPP $E$-field amplitude saturates due to the propagation loss.

\section{DESIGN AND SIMULATIONS}

In this section, in order to apply and validate the optimal launching condition, various SPPs launching structures made of nonuniform DRAs arrays on a metal surface are designed 




(a)

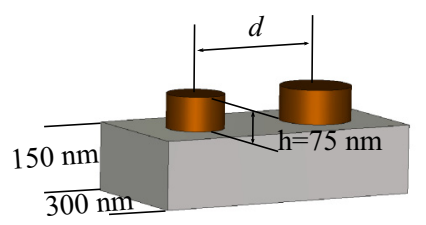

(b)

FIG. 9. (Color online) The designed SPP launcher. (a) Illustration for Cases 1 and 2 with a difference in the interelement spacing $d$ and resonator sizes. (b) Illustration for Case 3 as a bidirectional coupler.

and demonstrated through full-wave simulation. The designed structures are shown in Fig. 9. All dielectric resonators are cylindrical in shape with varying radii but a uniform height of $75 \mathrm{~nm}$. The center distance between neighboring resonators is denoted as $d$. Resonators are located on a silver substrate, which has a thickness of $150 \mathrm{~nm}$. $\mathrm{TiO}_{2}$ is selected for the dielectric resonators because of its electrical properties and relative ease of patterning at nanoscale. The material offers an anisotropic relative permittivity of 8.29 along the planar axes and 6.71 along the cylindrical axis with a loss tangent of less than 0.01 [34]. Silver, as the metal substrate, offers a relative permittivity of $-16.05+i 0.48$ at $633 \mathrm{~nm}$ [34]. At $633 \mathrm{~nm}$, the SPP wavelength on the silver-air interface equals to $\lambda_{\mathrm{spp}}=\lambda_{0} \sqrt{\left(\epsilon_{\mathrm{Ag}}+\epsilon_{\mathrm{air}}\right) / \epsilon_{\mathrm{Ag}} \epsilon_{\mathrm{air}}}=613 \mathrm{~nm}$.

Three illustrative cases are selected from the $v=0,1$ orders $L$ and $R$ lines, as indicated by the numbered blue dots in Fig. 4(a). These practically realizable cases are selected with consideration of the sizes of resonators, which determine the smallest interelement distance $d$, since neighboring resonators cannot be patterned too close to each other. The corresponding element spacing $d$ and required phase progressions are summarized in Table I. Case 1 is selected on the ideal launching line with $v=0$ in the nondeflection zone, while Case 2 is on the line $v=1$ in the deflection zone. According to Eq. (4), Case 2 promises a first-order $(m=1)$ diffraction at an angle of $48.6^{\circ}$ from the normal direction. Case 3 is at the intersection of the lines $v=0(L)$ and $v=1(R)$, and hence Case 3 should couple incident light into SPPs propagating toward two opposite directions, resulting in standing waves. In addition to the three cases above, a previously published DRA-based

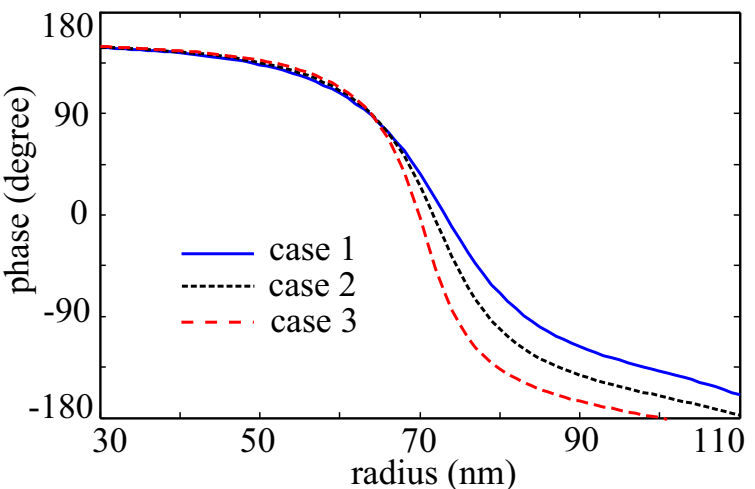

(a)

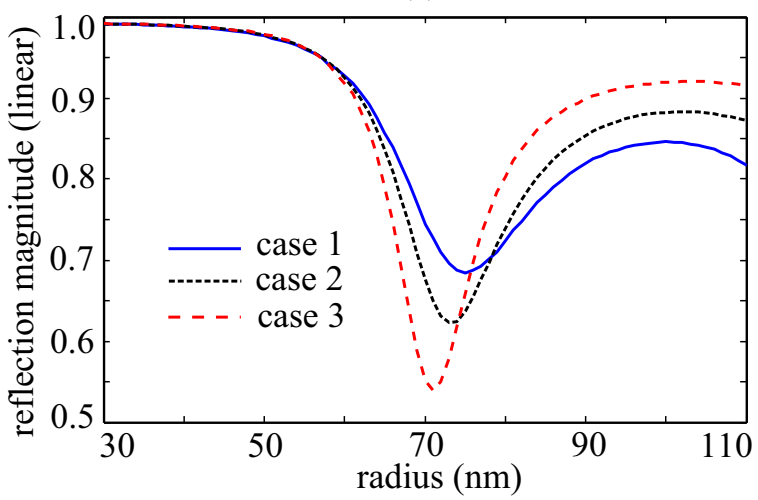

(b)

FIG. 10. (Color online) Numerically calculated phase responses (a) and reflection magnitudes (b) of unit cells corresponding to simulations of marked dots 1, 2, and 3 in Fig. 4(a).

reflectarray [34], labeled as Case 4, is also marked in Fig. 4(a). In this last case, the goal of the reflectarray design was deflection and thus the geometry is located far from the SPP launching lines so that most of the incident power is deflected at a predesigned offset angle from specular direction.

The required phase gradient for the three designs can be obtained by varying the size of the resonators. It can be shown that $\mathrm{TiO}_{2}$ resonators with different radii around resonance exhibit different phase responses and reflection magnitudes when illuminated normally by plane waves [34]. At $633 \mathrm{~nm}$, the phase curves and reflection magnitudes can be computed via numerical simulations in infinite uniform array conditions. This has to be done separately for the different interelement distance $d$ corresponding to the three considered cases, as presented in Fig. 10. The obtained phase curves cover a range of about $330^{\circ}$, which is commonly deemed sufficient

TABLE I. Design parameters for the examples denoted as numbered blue dots in Fig. 4(a).

\begin{tabular}{lcccc}
\hline \hline Case & 1 & 2 & 3 & $4[34]$ \\
\hline$\Delta \phi$ & $6 \pi / 7$ & $6 \pi / 7$ & $\pi$ & $\pi / 3$ \\
$d$ & $3 \lambda_{\text {spp }} / 7(265 \mathrm{~nm})$ & $4 \lambda_{\text {spp }} / 7(350 \mathrm{~nm})$ & $\lambda_{\text {spp }} / 2(300 \mathrm{~nm})$ & 2 \\
$N$ & 7 & 7 & $\lambda_{\text {spp }}(600 \mathrm{~nm})$ & 6 \\
Subarray length & $3 \lambda_{\text {spp }}(1855 \mathrm{~nm})$ & $4 \lambda_{\text {spp }}(2450 \mathrm{~nm})$ & Standing waves & $3 \lambda_{0}(1860 \mathrm{~nm})$ \\
Function & Left launching & Right launching & Deflection \\
\hline \hline
\end{tabular}


TABLE II. Radii of DRAs in the four featured simulation cases.

\begin{tabular}{lccccccc}
\hline \hline Case & \multicolumn{7}{c}{ Radius (nm) } \\
\cline { 2 - 8 } & $R_{1}$ & $R_{2}$ & $R_{3}$ & $R_{4}$ & $R_{5}$ & $R_{6}$ & $R_{7}$ \\
\hline 1 & 106 & 63.5 & 105.5 & 61.5 & 113 & 84.5 & 51.5 \\
2 & 84.5 & 69.5 & 99.5 & 58.5 & 35 & 82.5 & 57.5 \\
3 & 64 & 79 & $\#$ & $\#$ & $\#$ & $\#$ & $\#$ \\
$4[34]$ & 33 & 77 & 85 & 90 & 96.5 & 121 & $\#$ \\
\hline \hline
\end{tabular}

for practical designs. For the first three design cases marked by the blue dots in Fig. 4(a), the radii of the individual DRAs are selected from the phase curves to cover a cycle of $2 \pi$ phase change and the obtained values are summarized in Table II. These examples are specifically chosen to allow periodic repetition in subarrays formed by a small number of DRAs covering one phase cycle. The simulations are carried out with the frequency-domain solver of CST Microwave Studio, using unit cell boundary conditions and TM Floquet ports. Mutual coupling between the resonators with different sizes influences the local phase responses. Since the phases are calculated in a uniform array configuration, an optimization of DRAs radii is necessary to compensate nonuniform coupling and achieve optimal launching. The optimization is carried out for Cases 1 and 2 by maximizing the Poynting vector integrated on a series of selected planes normal to the silver surface [as represented in Fig. 9(a)]. In contrast, for simulation of Case 3, no optimization is required as the effect of DRA phase variation originating from nonuniform coupling is negligible.

Figure 11 shows for Cases 1, 2, 3, and 4 the simulated $E$-field component normal to the interface. This particular field component contains most features of the launched plasmonic waves and includes some features from the scattered field. As shown in Figs. 11(a) and 11(b) (corresponding movies available in the Supplemental Material [39]), both of the $\mathrm{TiO}_{2}$-based arrays for Cases 1 and 2 show clear directional SPP launching, with a distinctive difference in the scattered field. In addition to rightward directional launching, the result of Case 2 also shows a clear deflection wavefront at $48^{\circ}$, close to the theoretically calculated angle of $48.6^{\circ}$. In comparison, the result of Case 1 only shows leftward directional SPP launching and weak free-space scattering with no clearly defined direction. Furthermore, the simulations show that the accepted power, which includes power coupled into SPPs and power dissipated into the materials, are $66 \%$ for Case 1 and $46 \%$ for Case 2. Simulation of Case 3 shows no directional SPP launching but only the expected standing waves. In this case, $67 \%$ of the incident light power is reflected while the rest is coupled into standing waves and dissipated as heat in the silver and $\mathrm{TiO}_{2}$. This effect suggests (after optimization) a possible application of DRA arrays to perfect absorption. Case 4 is the simulation reproducing the reflectarray reported in Ref. [34] and the result shows clear deflection at $19.9^{\circ}$ offset from normal direction.

The 3D simulation results also reveal some effects not accounted for in the analysis. The nonuniform arrays of DRAs on the silver surface are not only excited by normal incident light, but also by their neighboring resonators. This parasitic

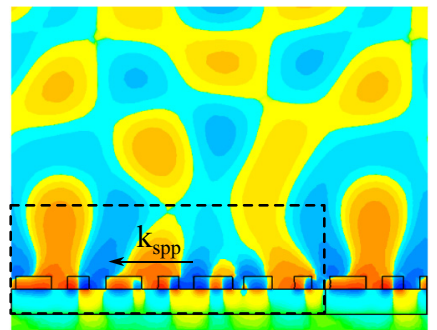

(a)

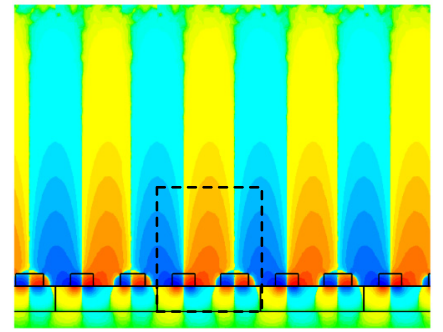

(b)

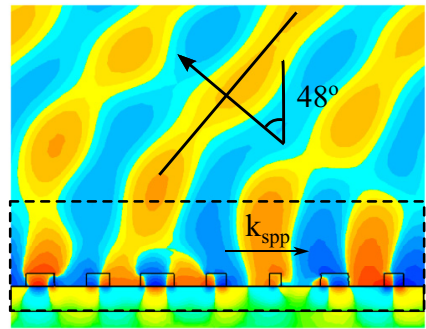

(c)
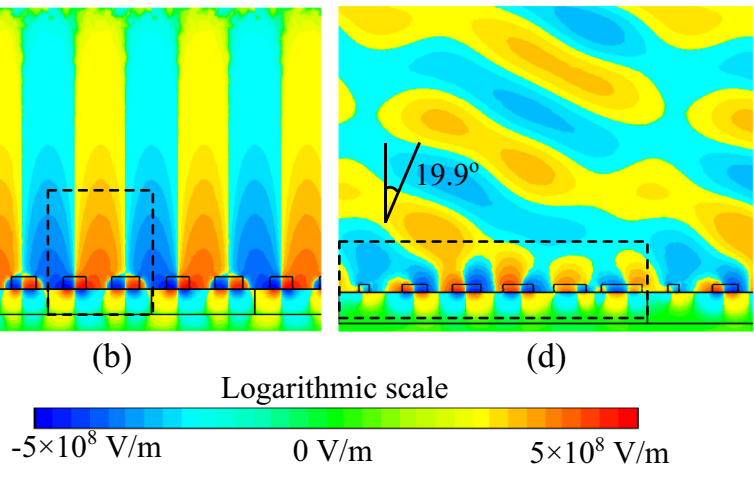

(d)

$5 \times 10^{8} \mathrm{~V} / \mathrm{m}$

FIG. 11. (Color online) Simulated $E$-field component perpendicular to the silver surface for the four cases indicated in Fig. 4(a). All cases share the same amplitude and geometry scale. The black dashed boxes indicate a single subarray. (a) Case 1: Leftward SPP launcher in the nondeflection zone. $34 \%$ of the incident power is reflected while $66 \%$ is accepted, including power coupled into SPPs and dissipated inside materials. (b) Case 2: Rightward SPP launcher in the deflection zone. $54 \%$ of the incident power is reflected while $46 \%$ is accepted. A deflection wavefront is visible at $48^{\circ}$ from the normal. (c) Case 3: A clear standing waves is observed on this structure with $67 \%$ of the incident power being reflected while $33 \%$ is absorbed. (d) Case 4: The reflectarray in Ref. [34] is reproduced without further optimization. A clear deflection is observed at $19.9^{\circ}$ off normal. $47 \%$ of the incident power is accepted mainly dissipated inside materials while $53 \%$ is reflected.

excitation through mutual coupling introduces an asymmetry [40] in the excited mode of each resonator. This effect can change the DRAs' phases and radiation patterns and as a result, higher-order modes are observed in some large DRAs. Therefore, the optimization procedure can yield DRA sizes that are dramatically different from the radii found directly from the phase curves computed in a uniform array configuration [Fig. 10(a)]. Furthermore, intrinsic differences in the elements' radiation magnitude also contribute to a difference observed between the analytical and numerical results. The resonant DRAs have larger scattering cross-section than off-resonance DRAs. This effect is more obvious when the progressive phase shift is large and can lead to stronger unwanted higher-order diffractions and negative impact on SPP launching.

\section{CONCLUSION}

SPP couplers based on nonuniform DRA arrays operating in their fundamental resonant magnetic dipole mode have been proposed. In a nonuniform array, a variation in the size of the DRAs can introduce a phase gradient to control 
the propagation direction of SPPs. By properly designing the SPP coupler array, optimal SPP launching can be achieved by avoiding energy leakage into deflection orders. The analysis presented in this article provides guidelines for designing efficient and compact SPP couplers.

\section{ACKNOWLEDGMENT}

C. Fumeaux acknowledges the Australian Research Council (ARC) Future Fellowship funding scheme for support under Grant No. FT100100585.
[1] S. A. Maier, Plasmonics: Fundamentals and Applications (Springer, Berlin, 2007).

[2] R. Charbonneau, N. Lahoud, G. Mattiussi, and P. Berini, Opt. Express 13, 977 (2005).

[3] D. Y. Fedyanin, A. V. Krasavin, A. V. Arsenin, and A. V. Zayats, Nano Lett. 12, 2459 (2012).

[4] S. A. Maier, Opt. Express 14, 1957 (2006).

[5] S. Wunderlich and U. Peschel, Opt. Express 21, 18611 (2013).

[6] A. J. Haes, C. L. Haynes, A. D. McFarland, G. C. Schatz, R. P. Van Duyne, and S. Zou, MRS Bull. 30, 368 (2005).

[7] J. Homola, S. Yee, and G. Gauglitz, Sensors Actuators B 54, 3 (1999).

[8] K. A. Willets and R. P. Van Duyne, Ann. Rev. Phys. Chem. 58, 267 (2007)

[9] S. Kawata, Y. Inouye, and P. Verma, Nat. Photon. 3, 388 (2009).

[10] N. Fang, H. Lee, C. Sun, and X. Zhang, Science 308, 534 (2005).

[11] D. Maystre, in Plasmonics: from Basics to Advanced Topics, edited by S. Enoch and N. Bonod (Springer, Berlin/Heidelberg, 2012).

[12] M. C. Hutley and D. Maystre, Opt. Commun. 19, 431 (1976).

[13] Q. Gan, Z. Fu, Y. J. Ding, and F. J. Bartoli, Opt. Express 15, 18050 (2007).

[14] Z. Fu, Q. Gan, K. Gao, Z. Pan, and F. J. Bartoli, J. Lightwave Technol. 26, 3699 (2008).

[15] F. López-Tejeira, S. G. Rodrigo, L. Martín-Moreno1, F. J. García-Vidal, E. Devaux, T. W. Ebbesen, J. R. Krenn, I. P. Radko, S. I. Bozhevolnyi, M. U. González, J. C. Weeber, and A. Dereux, Nat. Phys. 3, 324 (2007).

[16] J. Chen, Z. Li, S. Yue, and Q. Cong, Appl. Phys. Lett. 97, 041113 (2010).

[17] M. Kuttge, F. J. García de Abajo, and A. Polman, Opt. Express 17, 10385 (2009).

[18] L. Yin, V. K. Vlasko-Vlasov, A. Rydh, J. Pearson, U. Welp, S.-H. Chang, S. K. Gray, G. C. Schatz, D. B. Brown, and C. W. Kimball, Appl. Phys. Lett. 85, 467 (2004).

[19] A. Baron, E. Devaux, J. C. Rodier, J. P. Hugonin, E. Rousseau, C. Genet, T. W. Ebbesen, and P. L. Alanne, Nano Lett. 11, 4207 (2011).

[20] X. Huang and M. L. Brongersma, Nano Lett. 13, 5420 (2013).

[21] M. He, J. Liu, Z. Gong, S. Li, and Y. Luo, Opt. Commun. 285, $182(2012)$.

[22] J. Lin, J. P. Balthasar Mueller, Q. Wang, G. Yuan, N. Antoniou, X. Yuan, and F. Capasso, Science 304, 331 (2013).
[23] Y. Liu, S. Palomba, Y. Park, T. Zentgraf, X. Yin, and X. Zhang, Nano Lett. 12, 4853 (2012).

[24] Z. Xi, Y. Lu, W. Yu, P. Wang, and H. Ming, J. Opt. 16, 105002 (2014).

[25] C. Lu, X. Hu, H. Yang, and Q. Gong, Adv. Opt. Mater. 1, 792 (2013).

[26] L. Novotny and N. V. Hulst, Nat. Photon. 5, 83 (2011).

[27] P. Bharadwaj, B. Deutsch, and L. Novotny, Adv. Optics Photonics 1, 438 (2009).

[28] K. W. Leung, E. H. Lim, and X. S. Fang, Proc. IEEE 100, 2181 (2012).

[29] R. K. Mongia and P. Bhartia, in International Journal of Microwave and Millimeter-Wave Computer-Aided Engineering (John Wiley \& Sons, New York, 1994), Vol. 4(3), p. 230.

[30] Q. Lai, G. Almpanis, C. Fumeaux, H. Benedickter, and R. Vahldieck, IEEE Trans. Antennas Propag. 56, 3589 (2008).

[31] I. Staude, A. E. Miroshnichenko, M. Decker, N. T. Fofang, S. Liu, E. Gonzales, J. Dominguez, T. S. Luk, D. N. Neshev, I. Brener, and Y. S. Kivshar, ACS Nano 7, 7824 (2013).

[32] A. E. Krasnok, A. E. Miroshnichenko, P. A. Belov, and Y. S. Kivshar, Opt. Express 20, 20599 (2012).

[33] L. Zou, W. Withayachumnankul, C. M. Shah, A. Mitchell, M. Klemm, M. Bhaskaran, S. Sriram, and C. Fumeaux, IEEE Photon. J. 6, 4600110 (2014).

[34] L. Zou, W. Withayachumnankul, C. M. Shah, A. Mitchell, M. Bhaskaran, S. Sriram, and C. Fumeaux, Optical Express 21, 1344 (2013).

[35] Y. Yang, W. Wang, P. Moitra, I. I. Kravchenko, D. P. Briggs, and J. Valentine, Nano Lett. 14, 1394 (2014).

[36] L. Zou, M. López-García, W. Withayachumnankul, C. M. Shah, A. Mitchell, M. Bhaskaran, S. Sriram, R. Oulton, M. Klemm, and C. Fumeaux, Appl. Phys. Lett. 105, 191109 (2014).

[37] C. Palmer, Diffraction Grating Handbook, 6th ed. (Newport Corporation, Irvine, 2005).

[38] A. V. Zayats, I. I. Smolyaninov, and A. A. Maradudin, Phys. Rep. 408, 131 (2005).

[39] See Supplemental Material at http://link.aps.org/supplemental/ 10.1103/PhysRevB.91.085433 for gif file animations of the four designs in the paper.

[40] M. Neugebauer, T. Bauer, P. Banzer, and G. Leuchs, Nano Lett. 14, 2546 (2014). 